\title{
A note on logit choices in strategy transit assignment
}

\author{
Michael Florian • Isabelle Constantin
}

Received: 21 October 2011/Accepted: 12 April 2012/Published online: 9 May 2012

(C) Springer-Verlag + EURO - The Association of European Operational Research Societies 2012

\begin{abstract}
Since it was first developed [see Spiess and Florian, Transp Res 23:83-102 (1989)], the strategy-based transit assignment has been extensively used and its properties are well understood now. The computation of an optimal strategy is relatively fast and is comparable to the computation of a shortest path tree for one destination. However, since it is the solution of a linear program, it produces extremal solutions. As a consequence, the sensitivity analysis of strategy flows is not smooth. This work parallels the contribution of Nguyen et al. (Transp Sci 32:54-64 1998) who developed a logit choice of strategies following a basic idea due to Dial (Transp Res 5:88-111, 1971), in order to consider a larger variety of strategies by allowing walk choices at nodes of the transit network. Nevertheless, since the network representation used is different from the one used by Nguyen et al. (Transp Sci 32:54-64, 1998), the development is different. This modified logit strategy transit assignment algorithm was shown to produce more realistic results in dense transit networks where relatively short walks are required for access to attractive alternative transit paths. It also models better access from centroids representing large zones.
\end{abstract}

Keywords Transit assignment - Optimal strategies · Discrete choice models

\footnotetext{
M. Florian ( $\square)$

CIRRELT, University of Montreal, P.O. Box 6128, Station Centre-ville, Montreal, QC H3C 3J7, Canada

e-mail: mike@crt.umontreal.ca

M. Florian - I. Constantin

INRO, 376 Victoria Ave, Suite 200, Montreal, QC H3Z 1C3, Canada
} 


\section{Introduction}

Since it was first developed (see Spiess and Florian 1989), the strategy-based transit assignment has been extensively used and its properties are well understood. The computation of optimal strategies is relatively fast and is comparable to the computation of a shortest path tree for one destination.

The concept of a strategy is based on the assumptions that transit travelers possess knowledge of the transit services provided prior to undertaking a trip and the only information that they obtain once the trip is started is which transit line arrives next at the stop where they are waiting for a service. Therefore, they are able to determine and use efficient strategies (see Bouzaiene-Ayari et al. 2001). On a transit network, a strategy was characterized by Nguyen and Pallottino (1988) and Nguyen et al. (1998) as a hyperpath, which is a directed acyclic graph connecting origins to a destination.

The consideration of congestion in transit vehicles and increased waiting time at stops due to the fact that the first vehicle to arrive may not be boarded since it may not have any space available, motivated the development of transit equilibrium models. Spiess and Florian (1989) and Nguyen and Pallottino (1988) formulated such transit equilibrium models which consider only the discomfort aboard the vehicles and represent it with increasing cost functions. These models can be solved by using adaptations of the linear approximation method of Frank and Wolfe (1956). Wu et al. (1994) proposed a different algorithm for solving the same model. Bouzaiene-Ayari et al. (2001) extended the Wu et al. (1994) model to study existence, uniqueness and equilibrium under the assumption that transit lines are used in inverse proportion to the waiting time for each line. They made the assumption that travel times are given by strongly monotone cost functions.

The idea of a strategy was exploited in a different way by Hamdouch et al. (2004) in devising a route choice method in a transit network with fixed, rigid capacities. At each boarding node, a user sorts the transit lines in decreasing order of preference, and boards the first vehicle in this list whose residual capacity is non-zero. Since a user's position in the queue varies from day to day, the delay experienced is stochastic. This leads to an equilibrium problem where, at a solution, users are assigned to strategies that minimize their expected delay. This model is formulated as a variational inequality, with a cost mapping which is discontinuous and strongly asymmetric. They provide an algorithm for solving this model.

The purpose of this paper is more modest and focuses on the fact that, in transit networks that are not subject to congestion, the strategy-based transit route choice model is an extreme point of the feasible solution set. This extremal property leads to a very efficient solution algorithm. However, in some situations, it may be more appropriate to model the choices made by the transit riders in a richer way. The purpose of this paper is to present an extension of the optimal strategy model that includes a richer set of strategies, using a discrete choice component. The approach taken is to generalize the method of Nguyen et al. (1998), who developed an algorithm for computing logit choice of strategies, following a basic idea due to Dial (1971), by considering a larger variety of strategies: at each transit stop, a choice is allowed between walking to another stop or boarding a line at the stop. Such choices 
were not considered by Nguyen et al. (1998): at a stop, the lesser cost choice of boarding a transit vehicle or walking to another stop is chosen.

The paper is organized as follows. In the next section, a review of the optimal strategy model formulation and its extension is presented. Then, a numerical example is used to illustrate the desired set of choices. The additional information kept about non-optimal options is explained. The statement of the algorithm and its application to a simple numerical example follow. A discussion of practical applications and a short conclusion end the article.

\section{Attractive lines in an optimal strategy: a review}

Before the development of the extended logit strategy model and algorithm, a review of some notation that refers to the transit network and to the computed quantities in a strategy is necessary.

The transit network considered is modeled with nodes $N$ and $\operatorname{arcs} A$ that form a directed graph $G=(N, A)$. The transit line segments and walk links are represented by arcs; boarding and alighting arcs are used to represent activities at stops. Each arc $a \in A$ has two attributes, $t_{a}$ and $f_{a}$, which are the travel time and the frequency, respectively. Figure 1 describes the way in which arcs are used to represent the alighting and boarding activities at a stop, the travel on transit line segments and the possible walk between stops:

The notation used is as follows. The frequency of an on-board arc (or transit line segment) is denoted by $f_{a}$ and it is assumed that the segment inherits the frequency of its transit line; $t_{a}$ is the travel time on the line segment or the walk time on the link. The set of walk links is denoted $A^{w}, A^{w} \in A$, and the walk arcs which are outgoing from node $i$ are referred to as $a, a \in A_{i}^{w+}$. The set of arcs which belong to transit lines is denoted by $A^{t}, A^{t} \in A$. The optimal strategy is denoted by $\bar{A}$ and the

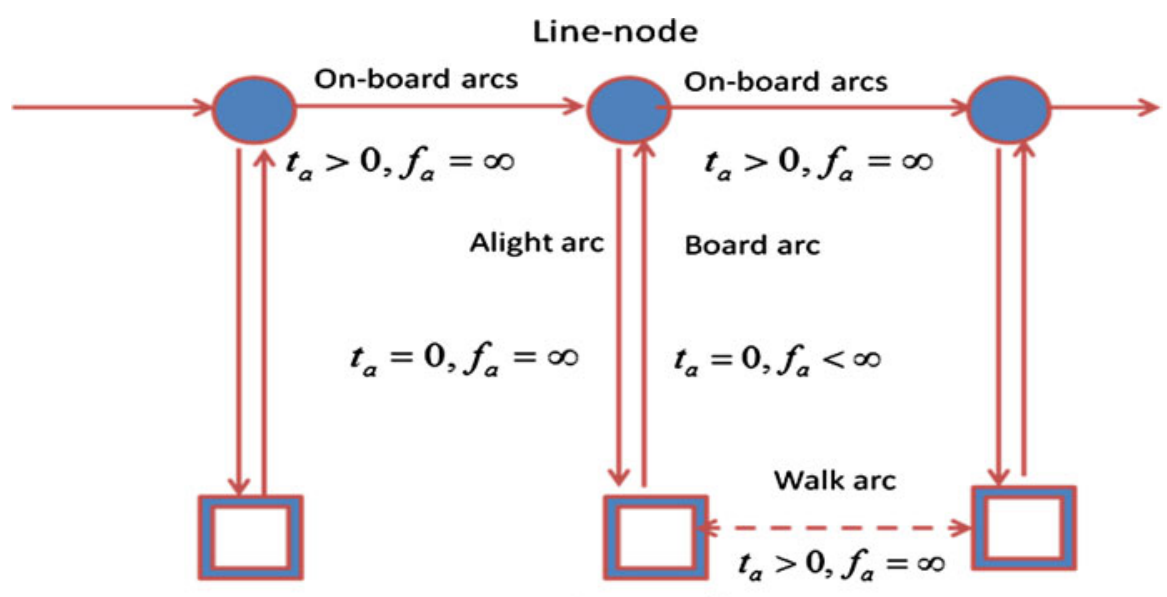

Stop-node

Fig. 1 The representation of lines and stops 
set of attractive lines (or the stops of the attractive lines) and walk links that are used at node $i$ is denoted $\overline{A_{i}}$. The optimal strategy at a node may consist of attractive lines or a single walk link. The combined frequency of the attractive lines at node $i$, if the attractive lines are part of the optimal strategy, is

$$
f_{i}=\sum_{a \in \bar{A}_{i}^{+}} f_{a},
$$

where $a$ denotes the arcs of attractive lines at node $i$. The proportion of trips that leave from stop (boarding) node $i$ on segment $a, a \in \bar{A}_{i}^{+}$, is

$$
p_{a}=f_{a} / \sum_{a^{\prime} \in \bar{A}_{i}^{+}} f_{a^{\prime}}, \quad a \in \bar{A}_{i}^{+} .
$$

The average waiting time at stop (boarding) node $i$ is

$$
w_{i}=1 / \sum_{a^{\prime} \in \bar{A}_{i}^{+}} f_{a^{\prime}} .
$$

For any on-board arc and walk arc $a$, there is a travel time $t_{a}$ and the flow proportion equals 1 .

Given travel demands $g_{i}^{d}, i \in N$, from all nodes $i$ to a single destination $d$, the optimization model that corresponds to finding the optimal strategy for a single destination is the linear program that seeks to find arc flows $v_{a}, a \in A$, that are the solution of

$$
\operatorname{Min} \sum_{a \in A} t_{a} v_{a}+\sum_{i} w_{i}
$$

Subject to

$$
\begin{gathered}
\sum_{a \in A_{i}^{+}} v_{a}-\sum_{a \in A_{i}^{-}} v_{a}=g_{i}^{d}, \quad i \in I \\
v_{a} \leq f_{a} w_{i}, \quad a \in A_{i}^{+}, i \in I, \\
v_{a} \geq 0, \quad a \in A .
\end{gathered}
$$

This model is applied to each destination $d \in D, D \subseteq I$, in turn, where $D$ is the set of all destinations. The algorithm used to compute the optimal strategy (from Spiess and Florian 1989) is given in the "Appendix".

In an optimal strategy, at each stop that has more than one transit line that can be boarded, an "attractive set" of transit lines is computed. Entire paths leading from the origin nodes to the destination can be recovered by analyzing the optimal strategy.

An intuitive interpretation of a strategy is that a transit rider makes chooses the route in a systematic way

1. Start the trip at a NODE. 
2. Board the vehicle that arrives first among the vehicles of the set of attractive lines at NODE.

3. Alight at the predetermined node.

4. If not yet at destination, set NODE to current node and return to step 1 . Otherwise, the trip is completed.

A numerical example that illustrates the difference between an optimal strategy and an enlarged choice set at nodes is presented next.

\section{A simple numerical example}

A simple example is shown in Fig. 2.

There are four transit lines that provide service from node $\mathrm{O}$ to node $\mathrm{D}$, which are not necessarily the initial origin and final destination of the trips. The line headways are given in the legend and the travel times are indicated on the transit line segments. The number of travelers that board at node $\mathrm{O}$ is 100 and they are all destined for $\mathrm{D}$. The volumes corresponding to the optimal strategy are shown on the links after the travel time in minutes. At node $\mathrm{O}$, there are 50 trips that board line 1 and alight at node D; the other 50 trips board line 2, alight at node B and then use lines 3 and 4 as shown in Fig. 2. The expected travel time from $\mathrm{O}$ to $\mathrm{D}$ is $27.75 \mathrm{~min}$ (note that the headway fraction was assumed to be 0.5 ).

Consider now the addition of another line that requires a walk from node $\mathrm{O}$ to node $\mathrm{E}$ in order to access line 5 which was added to the network. This is shown in Fig. 3.

The new line provides a total waiting and travel time of 26 min which is less than the $27.75 \mathrm{~min}$ of the optimal strategy before line 5 was added. Hence, the new optimal strategy for all 100 trips would be to walk to node E, board line 5 and alight at node D. None of the lines used before line 5 was added is used now as indicated in Fig. 4. All 100 trips will walk from node $\mathrm{O}$ to node $\mathrm{E}$ and then board line 5 to the destination $\mathrm{D}$.

On the other hand, if the travel time of line 5 would be 17 (instead of 15) minutes, then line 5 would receive no flow as indicated in Fig. 5.

It is this extremal property of the strategy transit route choice model that appears to be unreasonable in representing the behavior of transit travelers, which may not be quite as normative.

Now, suppose that a logit function applied to the expected waiting and travel times were to be used to make the choice between these two alternative strategies

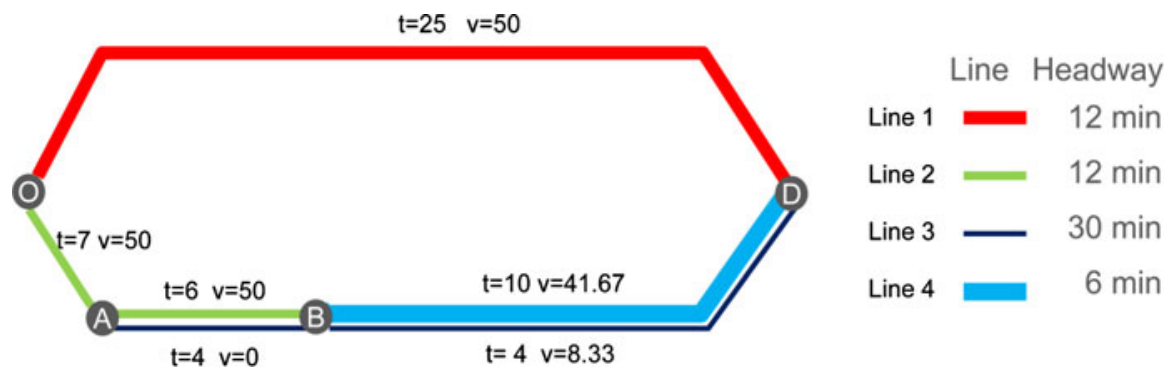

Fig. 2 A four-line example 


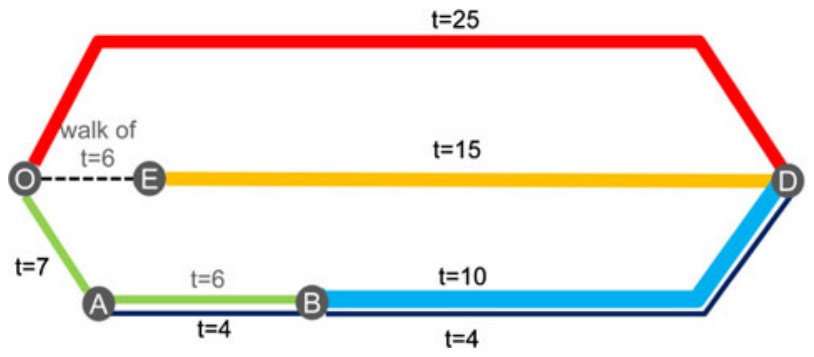

Line Headway

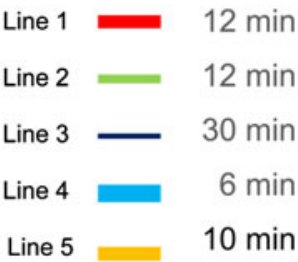

Fig. 3 A five-line example: adding a walk to line option

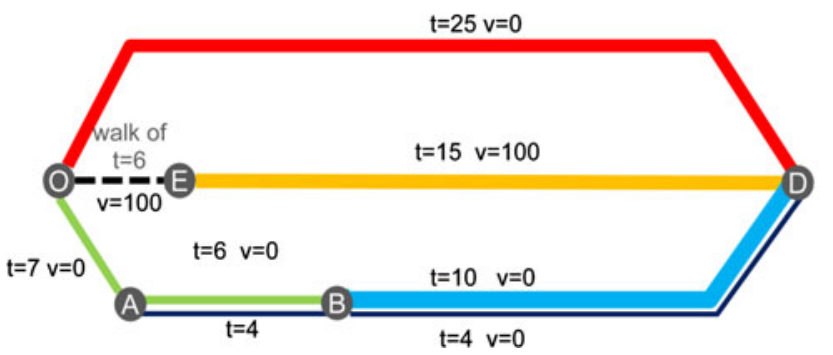

Line Headway

Line $1 \rightleftharpoons 12 \mathrm{~min}$

Line $2=12 \mathrm{~min}$

Line $3-30 \mathrm{~min}$

Line $4 \quad 6$ min

Line $5 \quad 10 \mathrm{~min}$

Fig. 4 A five-line example: walk to line strategy is optimal

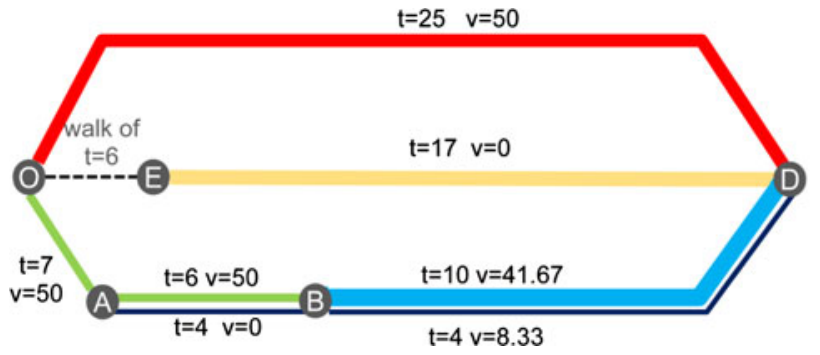

Line Headway

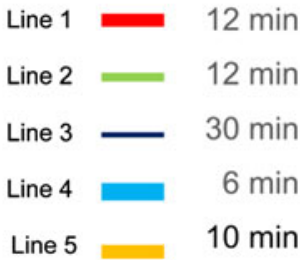

Fig. 5 A five-line example: waiting at $\mathrm{O}$ is optimal; walk to line 5 is not used

(one including the walk link and line 5; the other including all the lines except line 5). The probability (also referred to as the proportion) of the first strategy, which does not include line 5 , when the scale parameter $\theta=0.1$ would be:

$$
\exp (-\theta \times 27.75) /(\exp (-\theta \times 26)+\exp (-\theta \times 27.75))=0.458 .
$$

The resulting line segment flows are shown in Fig. 6. The logit function choice is applied to the two strategies; then for each strategy the flow subdivision on lines is done according to the proportions of the corresponding optimal or sub-optimal strategy. The new impedance is 26.80 , which is higher than 26 , since the resulting assignment is not optimal in the sense of least cost strategies. However, it may represent better the choices made by transit travelers.

One could envisage that more than one walk link may be used between two stops. Such an example is given below where a walk link, albeit a bit long, is used between 

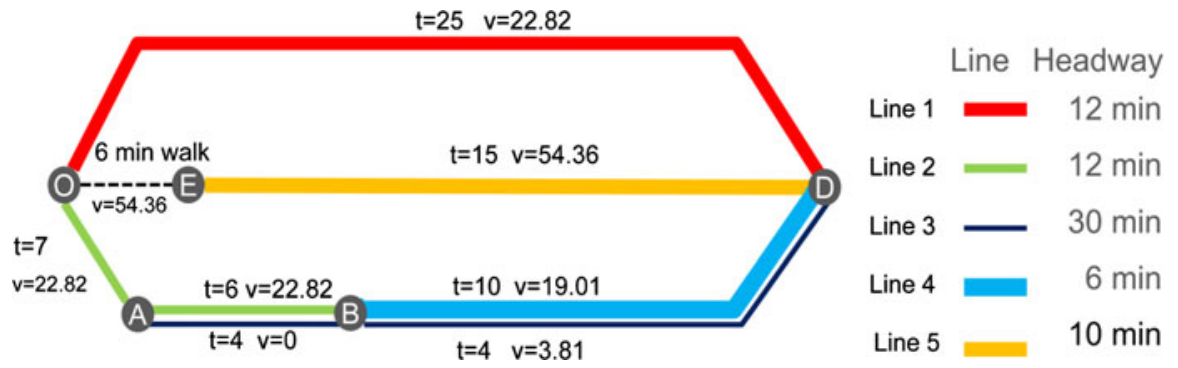

Fig. 6 A five-line example: logit choice of strategies
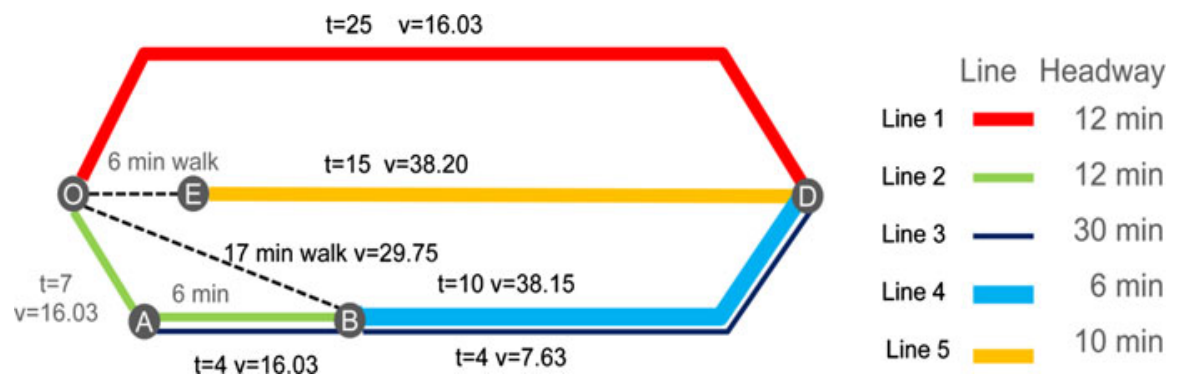

Fig. 7 A five-line example with two walk links: logit choice of strategies

nodes $\mathrm{O}$ and $\mathrm{B}$. The resulting flows, perhaps not entirely realistic, which are obtained by applying a logit model are shown in Fig. 7.

It is worthwhile to note that, in iterative transit equilibrium assignments (see Spiess and Florian 1989; Cepeda et al. 2006), where congestion in transit services is modeled, this extreme property of strategies would be attenuated: if the first strategy were to become congested, then the walk option would be part of the optimal strategy. In an equilibrium solution, both strategies are likely to be used.

As already mentioned, this development parallels the work by Nguyen et al. (1998). The difference between the latter model and this development is that walk was not considered as an option at a node (stop) in the construction of the strategies. The walk mode was only considered on connector links from an origin node, but not at intermediate nodes of the network, where there is a choice between boarding a vehicle at a stop and walking to another stop to board a different line.

To highlight the need for this development, it is useful to consider the following issue. Quite often, transit networks are coded with a single connector from an origin to the nodes of the transit network and there are no direct connector links to several transit services. Then the way to reach various services is via walk links, which lead to a boarding node. This is quite common with railway and metro/underground stations. As an example, consider the simple networks of Figs. 8 and 9, where there are three services: a bus service, a tram service, faster than bus, and a metro service, faster than both. There are 100 trips from origin node $\mathrm{P}$ to destination node Q. In Fig. 8, there are three connectors which lead directly to the three services and the 


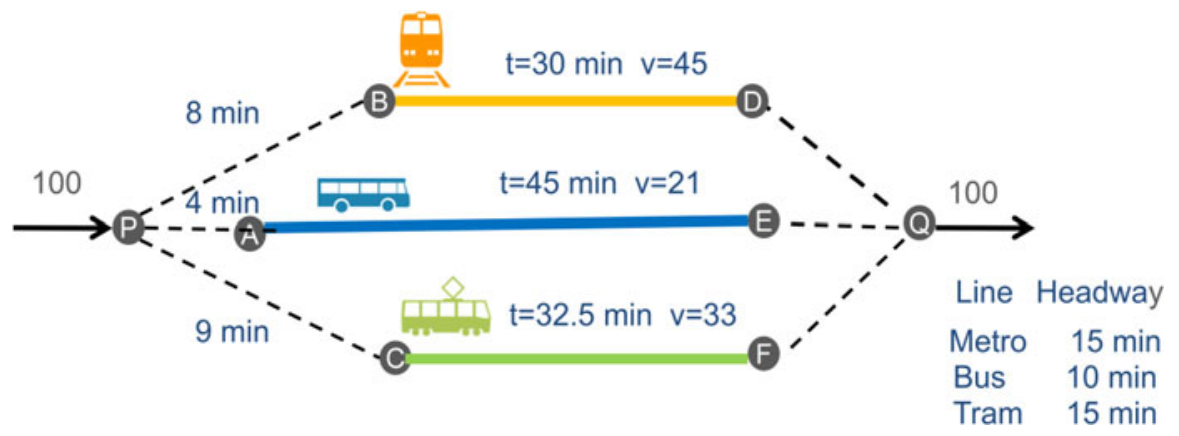

Fig. 8 A three-line example with direct connectors: logit choice of strategies at origin

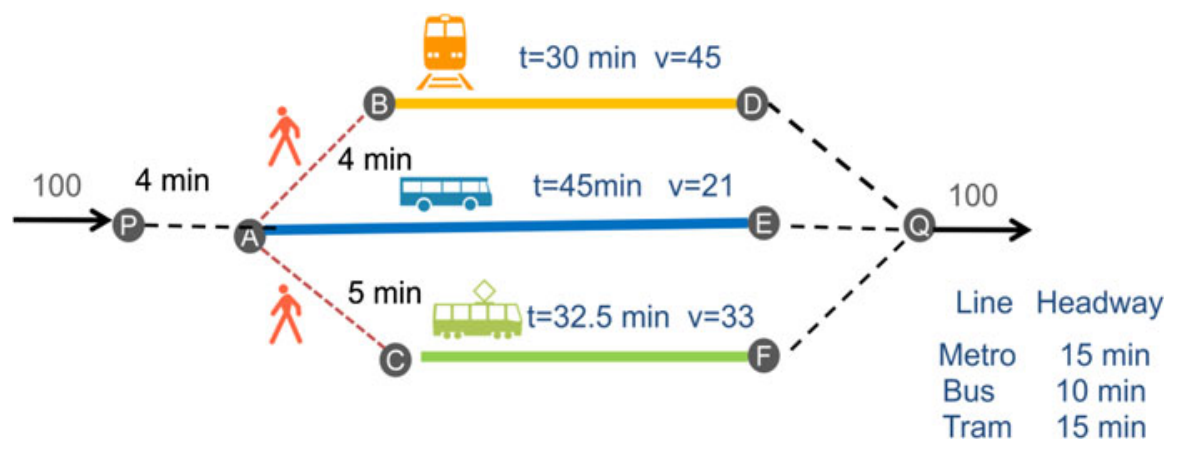

Fig. 9 A three-line example with one connector link and two walk links: logit choice of strategies at nodes

flows obtained by the logit model with a scale parameter of 0.2 are shown on the links.

In Fig. 9, there is a single connector to the node that is serviced by bus and walk links that lead to the tram and metro. The flows obtained with the logit on strategies model presented in this paper are the same as those in Fig. 8, since the walk links are considered. Otherwise, the Nguyen et al. (1998) method only provides the choice at origins among connector links and does not permit walk arcs. One can consider that this development generalizes the method to consider choices at all nodes and not only at origin nodes.

As the use of GIS maps increases, there is a tendency to code a single connector from an origin zone to the network and a relatively dense walk connects to transit stops. An example is given in Fig. 10. It is a part of the London network used by Transport for London for transit analyses.

The nodes highlighted with node numbers represent origins (zones) which are all connected to the street network with a single connector. The connectors do not all access transit stops directly as can be seen for instance for origins 1,500 and 1,540.

To consider such walk links in a logit choice of strategies, the modified Nguyen et al. (1998) model is described in the following. Then a solution algorithm is 


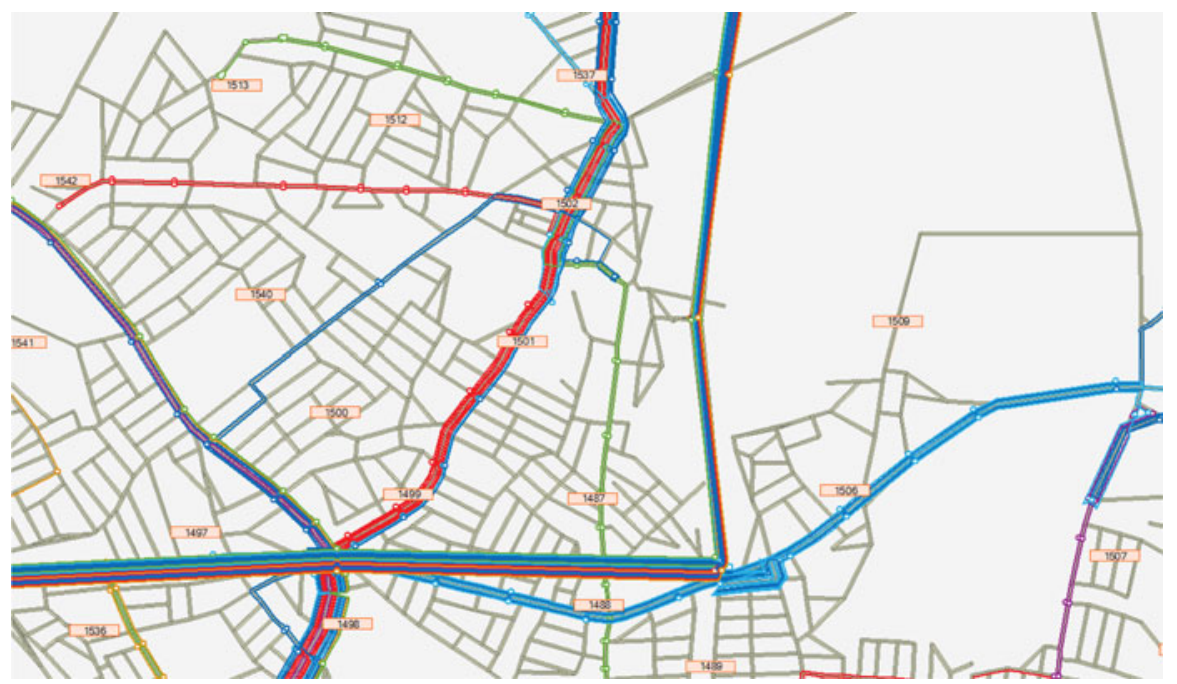

Fig. 10 Origins (zones) connected to a walk network (London)

developed in the subsequent sections. It uses accessibility measures by transit and by walk to each node of the network to model walk choices explicitly.

\section{Efficient arcs}

Clearly, one must first identify, in a very general way, the walk links and transit line segments that would be considered in an enlarged choice set of strategies. This leads to a modification of the algorithm that computes optimal strategies in order to save information about non-optimal choices in the computation of the optimal strategy. At a node, in the optimal strategy, either boarding a line from a set of attractive lines or using a single walk link is optimal. To make a choice between boarding a line at a stop or choosing among one or more walk links to another stop, there is a need to keep information about the non-optimal choices in addition to the optimal strategy.

Nguyen et al. (1998) used the basic idea of Dial (1971) to identify efficient arcs that should be considered. An efficient arc is considered loosely to be one that, if taken, leads nearer to the destination. This criterion is implemented by using the node labels computed in the optimal strategy algorithm. A walk arc $a=(i, j)$ is considered to be efficient if, in the solution of the model, the expected travel time to the destination from its tail node $i$ is larger than the travel time from its head node $j$.

$$
u_{i}>u_{j}
$$

In the context of the choice between transit and walk, the definition of an efficient arc adds to (8) the condition that no attractive line operates on the walk link. In a transit network where walking is allowed on all arcs, it is always better to ride than walk, assuming that the transit line speeds are much higher than the walk speed. Hence, let $a, a \in \tilde{A}_{i}^{w+}$ be the set of walk arcs that are efficient and do not have an attractive transit line which operates on the arc. 
There are two additional arc sets that are needed to apply the logit on strategies choice at a node: $\bar{A}_{i}^{w}$ and $\bar{A}_{i}^{t}$ which are, respectively, the optimal or best sub-optimal walk link at the node, and the optimal or best sub-optimal set of attractive transit lines at the node. The algorithm stated below builds the arc sets which are the best walk links and the best set of attractive lines for every node during the computation of the optimal strategy. For a given node $i$, the optimal strategy at the node is equal to one of these two sets.

\section{Algorithm I: Computing optimal strategies, best link set, best attractive line set}

Part 1: Find optimal strategy

1.1 (Initialization)

$$
\begin{aligned}
& u_{i}^{t}=\infty, i \in N-\{d\} ; u_{d}^{t}=0 ; \\
& u_{i}^{w}=\infty, i \in N-\{d\} ; u_{d}^{w}=0 ; \\
& f_{i}=0, i \in N ; \\
& S=A, \bar{A}=\phi, \bar{A}^{t}=\phi, \bar{A}^{w}=\phi .
\end{aligned}
$$

1.2 (Get next link) If $S=\phi$ then STOP,

Otherwise find $a=(i, j) \in S$ which satisfies

$$
\begin{aligned}
& u_{j}+t_{a} \leq u_{j^{\prime}}+t_{a^{\prime}}, a^{\prime}=\left(i^{\prime}, j^{\prime}\right) \in S ; \\
& S=S-(a) .
\end{aligned}
$$

1.3 (Update node labels) If $u_{i} \geq u_{j}+t_{a}$ then

$$
\begin{aligned}
& u_{i}=f_{i} u_{i}+f_{a}\left(u_{j}+t_{a}\right) /\left(f_{i}+f_{a}\right) \\
& f_{i}=f_{i}+f_{a}, \bar{A}=\bar{A}+\{a\}
\end{aligned}
$$

Obtain additional information for logit choice of strategies:

If $f_{a}<\infty$ then

include link as well in best attractive line set:

$$
u_{i}^{t}=u_{i}, \bar{A}^{t}=\bar{A}^{t}+\{a\} \text {, }
$$

Otherwise

$$
\text { include link as well in best link set }
$$

$$
u_{i}^{w}=u_{i}, \bar{A}^{w}=\bar{A}^{w}+\{a\} \text {, }
$$

Otherwise

Obtain additional information for logit choice of strategies :

If $f_{a}<\infty$ and $u_{i} \geq u_{j}$ and $u_{i}^{t} \geq u_{j}+t_{a}$ then

include link as well in best attractive line set :

$$
\begin{aligned}
& u_{i}^{t}=f_{i} u_{i}^{t}+f_{a}\left(u_{j}+t_{a}\right) /\left(f_{i}+f_{a}\right) \\
& f_{i}=f_{i}+f_{a}, \bar{A}^{t}=\bar{A}^{t}+\{a\},
\end{aligned}
$$

Go to step 1.2. 


\section{The algorithmic approach: node likelihoods and node proportions}

Next, the logit choice between transit and walk is considered. It is useful to recall that when using a logit path choice for highway networks, where the waiting time for vehicle services is not modeled, the flow assigned to each path $k$ is:

$$
p_{k}=\exp \left(-\theta C_{k}\right) / \sum_{k^{\prime} \in K_{\mathrm{od}}} \exp \left(-\theta C_{k^{\prime}}\right) \quad \text { for od } \in \mathrm{OD},
$$

where $p_{k}$ is the logit proportion (probability), $\theta$ is a scale parameter, $K_{\mathrm{od}}$ is the set of paths for origin-destination pair od and $C_{k}$ is the cost of path $k$. In all efficient algorithms that compute the logit path choice (see for instance Trahan 1974), an equivalent sequential form is used based on the conditional proportion:

$$
p_{(a \mid i)}=\exp \left(-\theta\left(t_{a}+T_{K(j)}^{\prime}\right)\right) / \sum_{a^{\prime} \in A_{i}^{+}} \exp \left(-\theta\left(t_{a^{\prime}}+T_{K\left(j^{\prime}\right)}^{\prime}\right)\right), \text { all } a=(i, j) \in A_{i}^{+}
$$

where $T_{K(j)}$ is defined as the $\log \operatorname{sum} T_{K(j)}=1 / \theta \ln L_{j}, L_{i}=\sum_{(i, j) \in A_{i}^{+}} \exp \left(-\theta t_{(i, j)}\right) L_{j}$, $t_{(i, j)}\left(=t_{a}\right)$ is the cost of arc $a$ and $A_{i}^{+}$is the set of arcs outgoing from node $i$ that satisfy $u_{i}>u_{j}$. In the case of road networks, the two methods produce the same proportions.

This is not necessarily the case for transit networks, due to the waiting costs which depend on the destination. The sequential method is adapted for the logit choice of strategies, since it does not require an explicit enumeration of all strategies and it can be integrated with the computation of the optimal strategies from destination to all origins in an efficient way. The difference lies in the way that common lines at a node are handled.

Once the sets $\bar{A}, \bar{A}^{w}, \bar{A}^{t}$, and $\tilde{A}_{i}^{w+}$ have been identified, the logit proportions are found by computing node likelihoods for all "efficient" walk links and transit line arcs. The node and arc likelihoods $L_{i}, L_{a}$ are computed recursively by a modification of the formulae used by Nguyen et al. (1998). To define these likelihoods, some additional notation must be introduced:

$L_{a}$ is the likelihood of arc $a$,

$L_{i}^{t}$ is the likelihood of the transit option at node $i$,

$L_{i}$ is the combined likelihood for node $i$,

$\tilde{t}_{a}$ is the sum of travel time for the sequence of on-board arcs from node $i$ to node $k(a)$, which is the optimal alighting node for arc $a=(i, j)$

The node and arc likelihoods are defined as follows:

a.

$$
L_{i}=1 \quad \text { if } i \text { is the destination; }
$$

b.

$$
\begin{aligned}
& L_{a}=\exp \left(-\theta \tilde{t}_{a}\right) \times L_{k(a)}, \text { if } a \in \bar{A}_{i}^{t+}, \\
& L_{a}=\exp \left(-\theta t_{a}\right) \times L_{j}, \text { if } a \in \tilde{A}_{i}^{w+}, \\
& \text { otherwise } L_{a}=0 ;
\end{aligned}
$$


c.

$$
L_{i}^{t}=\exp \left(-\theta w_{i}\right) \prod_{a \in \bar{A}_{i}^{t+}} L_{a}^{\left(f_{a} / f_{i}\right)}
$$

d.

$$
\begin{aligned}
& p_{i}^{t}=L_{i}^{t} /\left(L_{i}^{t}+\sum_{a \in \tilde{A}_{i}^{w+}} L_{a}\right), \\
& p_{a}=L_{a} /\left(L_{i}^{t}+\sum_{a \in \tilde{A}_{i}^{w+}} L_{a}\right)
\end{aligned}
$$

e.

$$
L_{i}=L_{i}^{t}+\sum_{a \in \tilde{A}_{i}^{w+}} L_{a}
$$

The node proportions and the node likelihoods can be computed simultaneously in a single scan of the network from destination to origins. The entire algorithm is stated next.

\section{Algorithm II: Computing logit choice walk/transit strategies}

1. Apply algorithm I for computing optimal strategies, best link set, best attractive line set. Also, for each node $i$ : if walk is better than transit $\left(u_{i}^{w}<u_{i}^{t}\right)$ and at least one attractive line operates on the optimal walk link, empty the set of attractive lines: $\bar{A}_{i}^{t+}=\phi$.

2. Compute likelihoods and proportions

For each node $i$ (processed in topological order):

- compute link likelihoods:

$$
\begin{aligned}
& L_{a}=\exp \left(-\theta \tilde{t_{a}}\right)^{*} L_{k(a)} \text { for each attractive arc } a, a \in \bar{A}_{i}^{t+} \\
& L_{a}=\exp \left(-\theta t_{a}\right)^{*} L_{j} \text { for each efficient walk arc } a, a \in \tilde{A}_{i}^{w+}
\end{aligned}
$$

- compute node transit likelihood based on the set of attractive lines:

$$
L_{i}^{t}=\exp \left(-\theta w_{i}\right) \prod_{a \in A_{i}^{+}} L_{a}^{\left(f_{a} / f_{i}\right)}
$$

if the set of attractive lines is empty $\left(\bar{A}_{i}^{t+}=\phi\right): L_{i}^{t}=0$

- compute transit proportion at node $i$ :

$$
p_{i}^{t}=L_{i}^{t} /\left(L_{i}^{t}+\sum_{a \in \tilde{A}_{i}^{w+}} L_{a}\right)
$$

- compute walk proportion for each efficient walk arc $a, a \in \tilde{A}_{i}^{w+}$ :

$$
p_{a}=L_{a} /\left(L_{i}^{t}+\sum_{a \in \tilde{A}_{i}^{w+}} L_{a}\right)
$$

- compute combined node likelihood:

$$
L_{i}=L_{i}^{t}+\sum_{a \in \tilde{A}_{i}^{w+}} L_{a}
$$


3. Load network

3.1 (Initialization) $\quad V_{i}=g_{i}, i \in N$

3.2 (Loading) $\quad$ For each node $i$ (processed in reverse topological order):

- compute transit flows for each attractive line segment $a \in \bar{A}_{i}^{t+}$ :

$$
v_{a}=p_{i}^{t} V_{i}\left(f_{a} / f_{i}\right) ; V_{j}=V_{j}+v_{a}
$$

- compute walk flows for each efficient walk arc $a, a \in \tilde{A}_{i}^{w+}$ :

$v_{a}=p_{a} V^{i} ; V_{j}=V_{j}+v_{a}$

The variable $V_{i}$ denotes the total flow that is leaving node $i$. The destination node is $d$.

\section{A numerical example}

The numerical example presented in the first section of the paper is used to demonstrate the algorithm. It has been constructed to show a simple example of the identification of the "efficient" segments and/or walk links and the application of the logit model to expand the choices at nodes where walk to a transit line is an attractive option (Fig. 11).

The labels $u_{i}^{t}, u_{i}^{w}$ are indicated in the dashed line boxes at nodes. The proportion of flow assigned to transit lines in the optimal strategy is indicated in the solid boxes on each link. The only node where there is a choice between transit and walk is node $\mathrm{O}$; in the optimal strategy, only the walk option, $\mathrm{O} \rightarrow \mathrm{E} \rightarrow \mathrm{D}$, is retained. However, for the logit strategy computation, transit line segments OD1 and OA2 make up the best attractive line set of node $\mathrm{O}$ :

OD1 is efficient since $u_{\mathrm{O}}=26>u_{\mathrm{D}}=0$

OA 2 is efficient since $u_{\mathrm{O}}=26>u_{\mathrm{B}}=11.5$

Note: alighting node $k(\mathrm{OA} 2)=B$

Next, the computation of the logit assignment is done by scanning the network from destination to origins (topological order) to compute recursively the link and node likelihoods as follows:

Node D: $u_{\mathrm{D}}=0$

$$
L_{\mathrm{D}}=1
$$

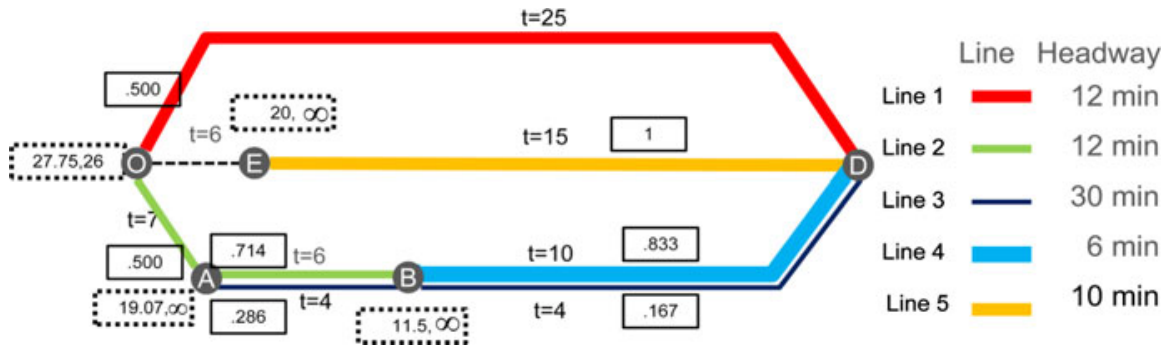

Fig. 11 Computation of the optimal strategy 
Node B: $u_{\mathrm{B}}=11.5$

$$
\begin{aligned}
L_{\mathrm{BD} 3} & =\exp (-0.1 \times 4) \times 1=0.67032 \\
L_{\mathrm{BD} 4} & =\exp (-0.1 \times 10) \times 1=0.367879 \\
L_{\mathrm{B}}^{t} & =\exp (-0.1 \times 2.5) \times 0.67032^{0.167} \times 0.367879^{0.833}=0.3167 \\
p_{\mathrm{B}}^{t} & =0.3167 / 0.3167=1 \\
L_{\mathrm{B}} & =0.3167^{1}=0.3167
\end{aligned}
$$

Node A: $u_{\mathrm{A}}=19.07$

$$
\begin{aligned}
L_{\mathrm{AB} 2} & =\exp (-0.1 \times 6) \times 0.3167=0.173809 \\
L_{\mathrm{AB} 3} & =\exp (-0.1 \times(4+4)) \times 1=0.449329 \text { Note: alighting node } k(\mathrm{AB} 3)=D \\
L_{\mathrm{A}}^{t} & =\exp (-0.1 \times 4.286) \times 0.173809^{0.714} \times 0.449329^{0.286}=0.148561 \\
p_{\mathrm{A}}^{t} & =0.148561 / 0.148561=1 \\
L_{\mathrm{A}} & =0.148561^{1}=0.148561
\end{aligned}
$$

Node E: $u_{E}=20$

$$
\begin{aligned}
L_{\mathrm{ED} 5} & =\exp (-0.1 \times 15) \times 1=0.22313 \\
L_{\mathrm{E}}^{t} & =\exp (-0.1 \times 5) \times 0.22313^{1}=0.135335 \\
p_{\mathrm{E}}^{t} & =0.135335 / 0.135335=1 \\
L_{\mathrm{E}} & =0.135335^{1}=0.135335
\end{aligned}
$$

Node O: $u_{O}=26$

$$
\begin{aligned}
L_{\mathrm{OD} 1} & =\exp (-0.1 \times 25) \times 1=0.082085 \\
L_{\mathrm{OA} 2} & =\exp (-0.1 \times(7+6)) \times 0.3167=0.086311 \text { Note: alighting node } k(\mathrm{OA} 2)=B \\
L_{\mathrm{OE}} & =\exp (-0.1 \times 6) \times 0.135335=0.074273 \\
L_{\mathrm{O}}^{t} & =\exp (-0.1 \times 3) \times 0.082085^{0.5} \times 0.086311^{0.5}=0.062356 \\
p_{\mathrm{O}}^{t} & =0.062356 /(0.062356+0.074273)=0.456389 \\
p_{\mathrm{OE}} & =0.074273 /(0.062356+0.074273)=0.543611 \\
L_{\mathrm{O}} & =0.062356^{0.456389} \times 0.074273^{0.543611}=0.068575
\end{aligned}
$$

The network volumes can then be computed by scanning the network again, this time from origins to destination (reverse topological order) as follows:

Initialize volumes:

$$
V_{\mathrm{O}}=100
$$

$V_{i}=0$ for all other nodes

$$
v_{a}=0 \text { for all arcs }
$$




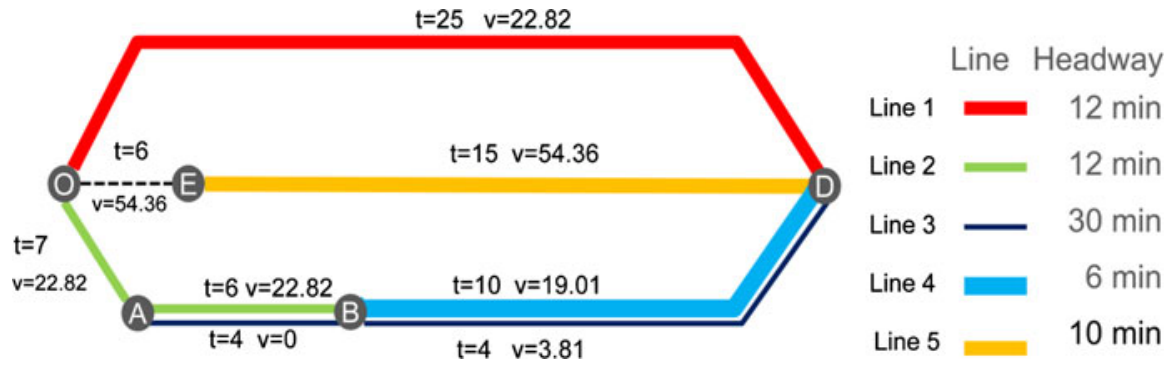

Fig. 12 Logit choice of strategies; the travel times and flows are indicated on the arcs

Node O: $V_{O}=100$

$$
\begin{aligned}
\mathrm{v}_{\mathrm{OD} 1} & =0+0.456389 \times 100 \times 0.5=22.82 \\
\mathrm{~V}_{\mathrm{D}} & =0+22.82=22.82 \\
\mathrm{v}_{\mathrm{OA} 2} & =0+0.456389 \times 100 \times 0.5=22.82 \\
\mathrm{v}_{\mathrm{AB} 2} & =0+22.82=22.82 \\
\mathrm{~V}_{B} & =0+22.82=22.82 \\
\mathrm{v}_{\mathrm{OE}} & =0.543611 \times 100=54.36 \\
\mathrm{~V}_{E} & =0+54.36=54.36
\end{aligned}
$$

Node E: $V_{E}=54.36$

$$
\begin{array}{r}
\mathrm{v}_{\mathrm{ED} 5}=0+1 \times 54.36 \times 1=54.36 \\
\mathrm{~V}_{\mathrm{D}}=22.82+54.36=77.18
\end{array}
$$

Node A: $V_{A}=0$

Node B: $V_{B}=22.82$

$$
\begin{aligned}
\mathrm{v}_{\mathrm{BD} 3} & =0+1 \times 22.82 \times 0.167=3.81 \\
\mathrm{~V}_{\mathrm{D}} & =77.18+3.81=80.99 \\
\mathrm{v}_{\mathrm{BD} 4} & =0+1 \times 22.82 \times 0.833=19.01 \\
\mathrm{~V}_{\mathrm{D}} & =80.99+19.01=100
\end{aligned}
$$

While the numerical example shows only a binary choice between "line" and "walk to line", any number of walk links may be considered (Fig. 12).

\section{Practical application}

The transit assignment algorithm described above has been implemented (see INRO 2010) and used in several applications. As an example, we show in Figs. 13 and 14 below the number of walk trips in central Brisbane, Australia for the optimal strategy and for the logit choice of strategies.

In the Brisbane network, the walk is allowed on all links in the central area of the city. A larger number of walk trips is consistent with the observations. In the 


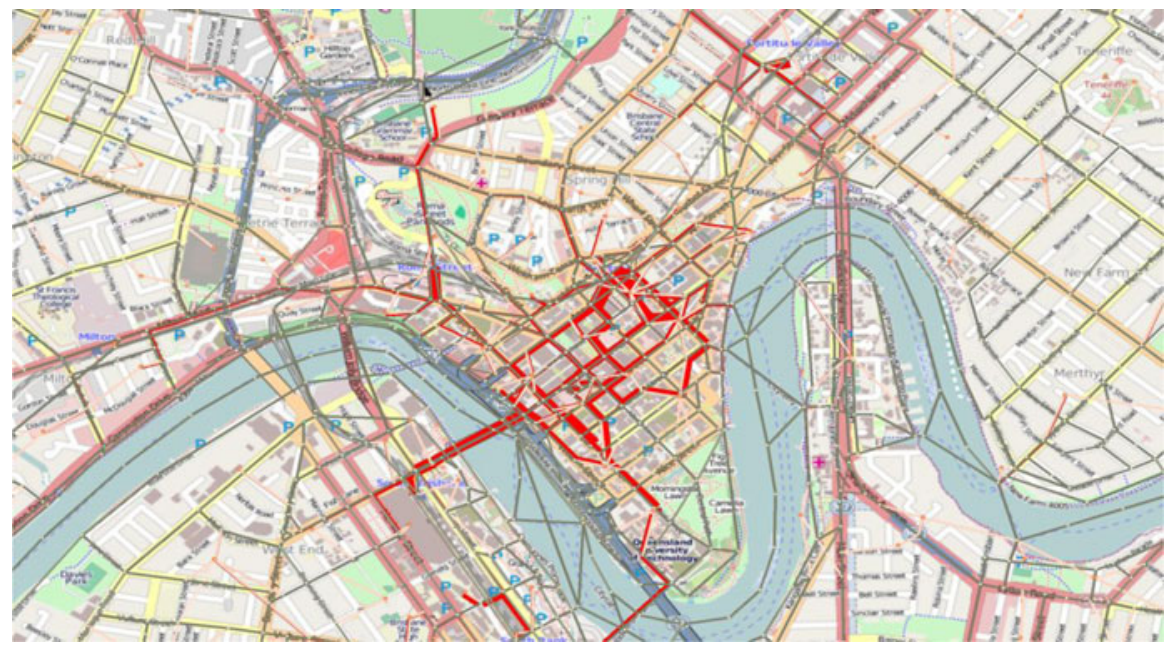

Fig. 13 Walk link flows for optimal strategy

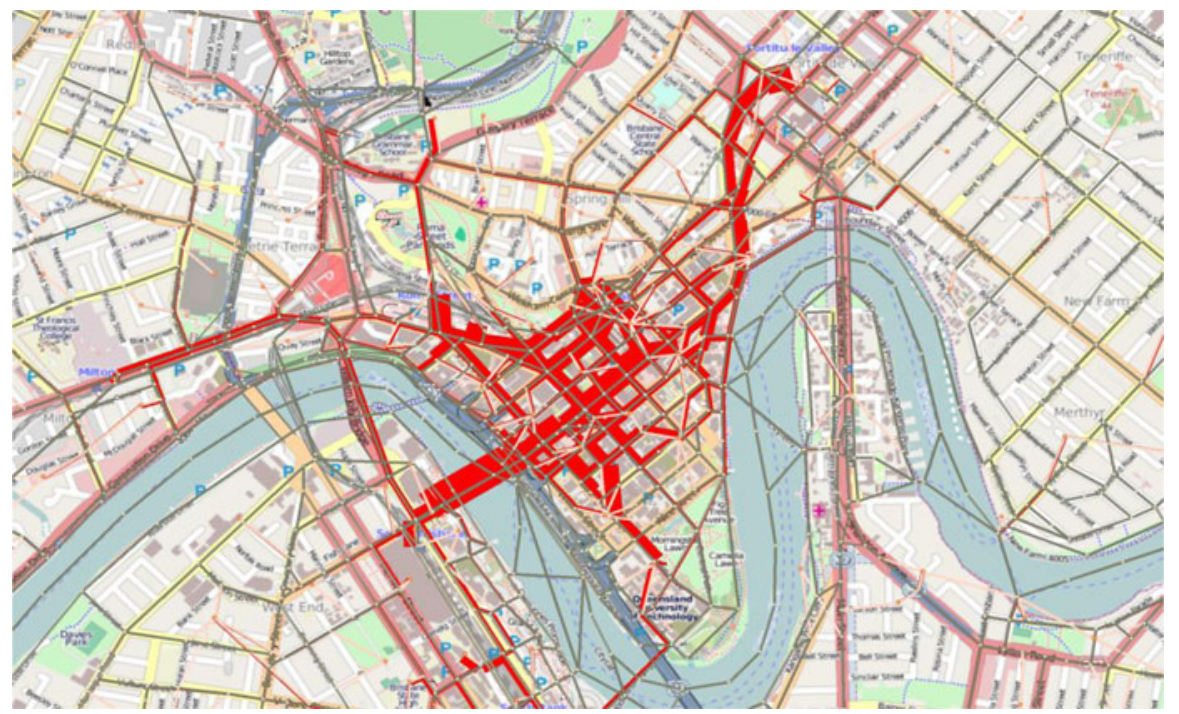

Fig. 14 Walk link flows for logit choice of strategies

optimal strategy, the walk trips are mostly, but not all, on the connector links. The logit on strategies walk volumes indicate a wider choice of boarding and alighting nodes than in the optimal strategy.

\section{Conclusions}

This development opens up new possibilities for better representing the choices that travelers make in transit route choices and is more flexible in application to different 
coding of walk links in a transit network model. One observation made in several applications is that the number of walk trips is increased by allowing a wider set of choices than that offered by the optimal strategy. On the other hand, the choices at connector links, if present, are consistent with the Nguyen et al. (1998) model. The calibration of the scale parameter of the logit function has not been addressed in this note, but it is a relevant issue. Another aspect that must be considered is the possible consequence of the IIA property of the logit function. In the applications carried out so far, no aberrant flows were observed. This is probably due to the fact that the logit function was used to choose among strategies. The selective application of the logit choice at certain nodes of the network, and not all, is advisable in practice.

\section{Appendix: Computing optimal strategies}

$u_{i}$ are node labels that contain, at the conclusion of the computations, the least cost travel and waiting times from node $i$ to the destination $d$.

\section{Part 1: Find optimal strategy}

1.1 (Initialization) $\quad u_{i}=\infty, i \in N-\{d\} ; u_{d}=0$;

$f_{i}=0, i \in N$;

$S=A ; \bar{A}=\phi$.

1.2 (Get next link) If $S=\phi$ then STOP,

Otherwise find $a=(i, j) \in S$ which satisfies

$$
\begin{aligned}
& u_{j}+t_{a} \leq u_{j^{\prime}}+t_{a^{\prime}}, a^{\prime}=\left(i^{\prime}, j^{\prime}\right) \in S ; \\
& S=S-\{a\} .
\end{aligned}
$$

1.3 (Update node label) If $u_{i} \geq u_{j}+t_{a}$ then

$$
\begin{aligned}
& u_{i}=f_{i} u_{i}+f_{a}\left(u_{j}+t_{a}\right) /\left(f_{i}+f_{a}\right) \\
& f_{i}=f_{i}+f_{a}, \bar{A}=\bar{A}+\{a\}
\end{aligned}
$$

go to step 1.2.

\section{Part 2: Assign demand according to optimal strategy}

2.1 (Initialization) $\quad V_{i}=g_{i}, i \in N$;

2.2 (Loading) $\quad$ Do for every link $a \in A$, in decreasing order of $\left(u_{j}+t_{a}\right)$;

$$
\text { if } \begin{aligned}
a \in \bar{A} \text { then } v_{a} & =V_{i} *\left(f_{a} / f_{i}\right), \\
V_{j} & =V_{j}+v_{a},
\end{aligned}
$$

otherwise $v_{a}=0$. 
The variable $V_{i}$ denotes the total flow that is leaving node $i$. At the beginning of the computations, the set $S$ includes all the links. The algorithm terminates when all the links are scanned. The convention $\infty \times 0=1$ has been used since, when the label of node $i, u_{i}$, is updated for the first time, $f_{i} u_{i}=\infty \times 0$. The solution of this linear program may be interpreted as a directed acyclic graph which Nguyen and Pallottino (1988) denoted to be a hyperpath.

The development of the algorithm that allows a logit choice of strategies by considering both walk options and a transit option at every node, when available, is based on a modification of the above algorithm.

\section{References}

Bouzaiene-Ayari B, Gendreau M, Nguyen S (2001) Modeling bus stops in transit networks: a survey and new formulations. Transp Sci 35(3):304-321

Cepeda M, Cominetti R, Florian M (2006) A frequency-based assignment model for congested transit networks with strict capacity constraints: characterization and computation of equilibria. Transp Res Part B 40(6):437-459

Dial RB (1971) A probabilistic multipath traffic assignment model which obviates path enumeration. Transp Res 5:88-111

Frank M, Wolfe P (1956) An algorithm for quadratic programming. Naval Res Logist Q 3:95-110

Hamdouch Y, Marcotte P, Nguyen S (2004) Capacitated transit assignment with loading priorities. Math Program B 101:205-230

INRO (2010) Emme 3 at http://www.inrosoftware.com

Nguyen S, Pallottino S (1988) Equilibrium traffic assignment in large scale transit networks. Eur J Oper Res 37(2):176-186

Nguyen S, Pallottino S, Gendreau M (1998) Implicit enumeration of hyperpaths in a logit model for transit networks. Transp Sci 32(1):54-64

Spiess H, Florian M (1989) Optimal strategies: a new assignment model for transit networks. Transp Res B 23(2):83-102

Trahan M (1974) Probabilistic assignment: an algorithm. Transp Sci 8(4):311-320

Wu JH, Florian M, Marcotte P (1994) Transit equilibrium assignment: a model and solution algorithms. Transp Sci 28(3):193-203 